\title{
Third-Order Neutral Delay Differential Equations: New Iterative Criteria for Oscillation
}

\author{
Osama Moaaz ${ }^{1 D},{ }^{1}$ Emad E. Mahmoud, ${ }^{2,3}$ and Wedad R. Alharbi ${ }^{4}$ \\ ${ }^{1}$ Department of Mathematics, Faculty of Science, Mansoura University, 35516 Mansoura, Egypt \\ ${ }^{2}$ Department of Mathematics and Statistics, College of Science, Taif University, P.O. Box 11099, Taif 21944, Saudi Arabia \\ ${ }^{3}$ Department of Mathematics, Faculty of Science, Sohag University, Sohag 82524, Egypt \\ ${ }^{4}$ Physics Department, Faculty of Science, University of Jeddah, Jeddah, Saudi Arabia
}

Correspondence should be addressed to Osama Moaaz; o_moaaz@mans.edu.eg

Received 27 October 2020; Revised 16 November 2020; Accepted 1 December 2020; Published 12 December 2020

Academic Editor: Kottakkaran Sooppy Nisar

Copyright (C) 2020 Osama Moaaz et al. This is an open access article distributed under the Creative Commons Attribution License, which permits unrestricted use, distribution, and reproduction in any medium, provided the original work is properly cited.

This study is aimed at developing new criteria of the iterative nature to test the oscillation of neutral delay differential equations of third order. First, we obtain a new criterion for the nonexistence of the so-called Kneser solutions, using an iterative technique. Further, we use several methods to obtain different criteria, so that a larger area of the models can be covered. The examples provided strongly support the importance of the new results.

\section{Introduction}

This study is concerned with developing new iterative criteria to test the oscillation of solutions of neutral delay differential equations NDDE of third order:

$$
\frac{\mathrm{d}}{\mathrm{d} l}\left(r(l)\left(\frac{\mathrm{d}^{2}}{\mathrm{~d} l^{2}} z(l)\right)^{\alpha}\right)+q(l) x^{\alpha}(\sigma(l))=0,
$$

where $l \geq l_{0}, z(l):=x(l)+p(l) x(\tau(l))$ is a corresponding function of $x, \alpha$ is a quotient of odd positive integers, $r \in C^{1}\left(T_{l_{0}}\right.$, $(0, \infty)), \int_{l_{0}}^{\infty} r^{-1 / \alpha}(s) \mathrm{d} s \longrightarrow \infty$ as $l \longrightarrow \infty, p \in C\left(T_{l_{0}},\left[0, p_{0}\right]\right)$, $p_{0}<1$ is a positive real number, $q \in C\left(T_{l_{0}},[0, \infty)\right), \tau, \sigma \in C^{1}$ $\left(T_{l_{0}}, \mathbb{R}\right), \quad \tau(l)<l, \quad \sigma(l)<l, \quad \lim _{l \rightarrow \infty} \tau(l)=\lim _{l \rightarrow \infty} \sigma(l)=\infty$, and $T_{l_{0}}:=\left[l_{0}, \infty\right)$.

By a solution of (1), we mean a nontrivial real function $x \in C\left(T_{l_{x}}, \mathbb{R}\right)$ for all $l_{x} \geq l_{0}$, which has the properties $z, r_{1} z^{\prime}$, and $r_{2}\left(r_{1} z^{\prime}\right)^{\prime} \in C^{1}\left(T_{l_{x}}, \mathbb{R}\right)$, and satisfies (1) on $T_{l_{x}}$. We only consider those solutions of (1) which exist on some halfline $T_{l_{x}}$ and satisfy the condition $\sup \left\{|x(l)|: l \geq l_{*}\right\}>0$ for any $l_{*} \in T_{l_{x}}$.
If the solution $x$ is either ultimately positive or ultimately negative, then $x$ is called nonoscillatory; otherwise, it is called an oscillatory solution. The equation itself is termed oscillatory if all its solutions oscillate. Solutions $x$ whose corresponding function $z$ satisfies $z(l) z^{\prime}(l)<0$ are called Kneser solutions. We denote the class of all Kneser solutions of (1) with the symbol $X_{K}$. Otherwise, $X_{N}$ denote to the class of all positive solutions of (1) whose $z$ satisfies $z(l) z^{\prime}(l)>0$.

Delay differential equations as a subclass of functional differential equations take into account the dependence on the system's past history, which results in predicting the future in a more reliable and efficient way. Neutral delay differential equations arise in various phenomena including problems concerning electric networks containing lossless transmission lines (as in high-speed computers where such lines are used to interconnect switching circuits), in the study of vibrating masses attached to an elastic bar or in the solution of variational problems with time delays, or in the theory of automatic control and in neuromechanical systems in which inertia plays a major role, see [1-6].

For interesting methods, techniques, and results which are concerned with the study of oscillation of third-order NDDEs, we refer the reader to the works [7-16]. Furthermore, the 
studies [17-20] are concerned with the study of odd-order DDEs.

At the beginning of any study of the oscillatory properties of solutions of differential equations, it is easy to notice the importance of classifying signs of derivatives of nonoscillatory solutions. For the positive solutions, based on the canonical condition $\int_{l_{0}}^{\infty} r^{-1 / \alpha}(s) \mathrm{d} s \longrightarrow \infty$ as $l \longrightarrow \infty$, it follows from ([21], Lemma 1) that $\left(r_{1} z^{\prime}\right)^{\prime}>0$ and there are two possible cases for $z^{\prime}(l)$ : either $z^{\prime}(l)>0$ or $z^{\prime}(l)<0$. By creating conditions that ensure $X_{N}$ and $X_{K}$ are empty sets, we can directly set the criteria for oscillation.

There are numerous results interested in finding conditions that ensure class $X_{N}$ is empty, which include Hille and Nehari types and Philos type. Baculikova and Dzurina [7] established a condition of Hille and Nehari type and proved that if

$$
\liminf _{l \rightarrow \infty} \int_{l}^{\infty} q(s) \frac{\sigma^{2 \alpha}(s)}{s^{\alpha}} \mathrm{d} s>\frac{(2 \alpha)^{\alpha}}{(\alpha+1)^{\alpha+1}\left(1-p_{0}\right)^{\alpha}},
$$

where $p_{0}<1$ and $r^{\prime}(l)>0$, then $X_{N}=\varnothing$. By comparison principles, Baculikova and Dzurina [8] proved that if the first-order DDE

$$
y^{\prime}(l)+q(l)\left(\frac{\left(\sigma(l)-l_{0}\right)^{2}(1-p(\sigma(l)))}{2 r^{1 / \alpha}(l)}\right)^{\alpha} y(\sigma(l))=0,
$$

is oscillatory, then $X_{N}=\varnothing$. We can easily notice that the delay argument $\tau(l)$ has been neglected in (2) and (3). Otherwise, by using the Riccati transformation, Thandapani and Li [16] guaranteed that class $X_{N}$ is empty if

$$
\limsup _{l \rightarrow \infty} \int_{l_{0}}^{l}\left(\frac{1}{2^{\alpha-1}} \rho(s) Q(s)-\frac{\left(1+p_{0}^{\alpha} / \tau_{0}\right)}{(\alpha+1)^{\alpha+1}} \frac{\left(\rho^{\prime}(s)\right)^{\alpha+1}}{\left(\rho(s) \beta_{1}\left(\sigma(s), l_{0}\right) \sigma^{\prime}(s)\right)^{\alpha}}\right)=\infty,
$$

where $\sigma^{\prime}(l)>0, \quad \tau^{\prime}(l) \geq \tau_{0}>0, Q(l):=\min \{q(l), q(\tau(l))\}$, and $\rho \in C\left(\left[l_{0}, \infty\right),(0, \infty)\right)$. All previous results focused on the class $X_{N}$ only and proved that every solution that belongs to $X_{K}$ tends to zero.

On the other hand, by establishing conditions for the nonexistence of Kneser solutions $\left(X_{K}=\varnothing\right)$, Dzurina et al. [12] attained the oscillation of all solutions of (1) in the linear case $\alpha=1$. They proved that if (4) and

$\liminf _{l \rightarrow \infty} \int_{\tau^{-1}(\rho(l))}^{l} Q(h)\left(\int_{\sigma(h)}^{\rho(h)} \int_{s}^{\rho(h)} \frac{1}{r(u)} \mathrm{d} u \mathrm{~d} s\right) \mathrm{d} h>\frac{\tau_{0}+p_{0}}{\tau_{0} \mathrm{e}}$,

hold, then equation (1) is oscillatory, where $\rho \in C\left(\left[l_{0}, \infty\right),(0\right.$

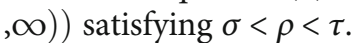

One purpose of this study is to further complement and improve the well-known results reported in the literature. In Section 2, by using an iterative technique, we get analogous iterative estimates for Kneser solutions of (1). These iterative estimates enable us to establish new criteria that ensure the nonexistence of Kneser solutions. Further, criteria of an iterative nature help check the oscillation, even when the other criteria fail to apply. In Section 3, we use the Riccati transformation method and comparison principles to obtain different criteria which guarantee that $X_{N}=\varnothing$. Examples illustrating the new results are also given.

For the sake of ease and assistance in presenting the main results, we provide the following notations and lemmas:

$$
\begin{aligned}
\theta(v, u) & :=\int_{u}^{v} r^{-1 / \alpha}(h) \mathrm{d} h, \text { for } u<v, \\
\delta & :=\left\{l l 1 \text { for } 0<\alpha \leq 1 ; 2^{1-\alpha} \text { for } \alpha>1 .\right.
\end{aligned}
$$

Lemma 1 (see [7], Lemma 1). All eventually positive solutions $x$ of (1) have the following properties:

$(\mathbf{P}) z$ and $z^{\prime \prime}$ are positive, $z^{\prime}$ is of fixed sign, and $r(l)$ $\left(z^{\prime \prime}(l)\right)^{\alpha}$ is nonincreasing, for l large enough.

Lemma 2 (see [16]). Let $\phi, \varphi \in[0, \infty)$. Then, $\delta(\phi+\varphi)^{\alpha} \leq\left(\phi^{\alpha}\right.$ $\left.+\varphi^{\alpha}\right)$ for all $\alpha \geq 0$.

\section{Main Results 1: Iterative Technique}

Lemma 3. Assume that $x$ belongs to $X_{K}$ and there is a function $\rho \in C\left(T_{l_{0}},(0, \infty)\right)$ with the property

$$
\sigma(l)<\rho(l)<\tau(l) .
$$

If $\tau^{-1}(u) \leq v$, then

$$
z(u) \geq \Theta_{n}(v, u) r^{1 / \alpha}(v) z^{\prime \prime}(v),
$$

for $n=0,1, \cdots$, where $\Theta_{0}(v, u):=\int_{u}^{v} \theta(v, h) \mathrm{d} h, Q(l):=\min$ $\{q(\tau(l)), q(l)\}$, and

$$
\begin{aligned}
\Theta_{n+1}(v, u):= & \int_{u}^{v} \int_{u_{2}}^{v}\left(\frac { 1 } { r ( u _ { 1 } ) } \operatorname { e x p } \left(\frac{\delta \tau_{0}}{\tau_{0}+p_{0}^{\alpha}} \int_{\tau^{-1}\left(u_{1}\right)}^{v}\right.\right. \\
& \left.\cdot Q(h) \Theta_{n}^{\alpha}(\rho(h), \sigma(h)) \mathrm{d} h 0\right)^{1 / \alpha} \mathrm{d} u_{1} \mathrm{~d} u_{2} .
\end{aligned}
$$

Proof. Suppose $x$ belongs to $X_{K}$. Thus, there is a $l_{1} \geq l_{0}$ satisfying $x(l)$, and $x(\sigma(l))$ and $x(\tau(l))$ are positive for $l \geq l_{1}$. As a direct result of Lemma $1, x$ achieves property $(\mathbf{P})$. 
Using induction, we will prove the iterative relationship (8).

$$
\begin{aligned}
& \text { At } n=0 \text {, since }\left(r(l)\left(z^{\prime \prime}(l)\right)^{\alpha}\right)^{\prime} \leq 0 \text {, we obtain } \\
& \qquad \begin{array}{l}
\int_{u}^{v} r^{-1 / \alpha}(h)\left(r(h)\left(z^{\prime \prime}(h)\right)^{\alpha}\right)^{1 / \alpha} \mathrm{d} h \\
\geq r^{1 / \alpha}(v) z^{\prime \prime}(v) \theta(v, u), \text { for } u<v,
\end{array}
\end{aligned}
$$

which in turn leads to

$$
-z^{\prime}(u) \geq z^{\prime}(v)-z^{\prime}(u) \geq r^{1 / \alpha}(v) z^{\prime \prime}(v) \theta(v, u) \text {, for } u<v \text {. }
$$

As a result of integrating (11) over $[u, v)$, we get

$$
\begin{aligned}
z(u) & \geq z(u)-z(v) \geq r^{1 / \alpha}(v) z^{\prime \prime}(v) \int_{u}^{v} \theta(v, h) \mathrm{d} h \\
& \geq \Theta_{0}(v, u) r^{1 / \alpha}(v) z^{\prime \prime}(v) .
\end{aligned}
$$

Next, we will prove (8) at $n+1$ based on the assumption that it is correct at $n$, that is,

$$
z(u) \geq \Theta_{n}(v, u) r^{1 / \alpha}(v) z^{\prime \prime}(v)
$$

First, we have from (1) that

$$
\begin{aligned}
q(\tau(l)) p_{0}^{\alpha} x^{\alpha}(\tau(\sigma(l))) & =q(\tau(l)) p_{0}^{\alpha} x^{\alpha}(\sigma(\tau(l))) \\
& =-\frac{p_{0}^{\alpha}}{\tau^{\prime}(l)}\left(r(\tau(l))\left(z^{\prime \prime}(\tau(l))\right)^{\alpha}\right)^{\prime} \\
& \leq-\frac{p_{0}^{\alpha}}{\tau_{0}}\left(r(\tau(l))\left(z^{\prime \prime}(\tau(l))\right)^{\alpha}\right)^{\prime} .
\end{aligned}
$$

By exploiting Lemma 2 with $\phi=x(l)$ and $\varphi=p_{0} x(\tau(l))$, we obtain

$$
\delta z^{\alpha}(l) \leq \delta\left(x(l)+p_{0} x(\tau(l))\right)^{\alpha} \leq x^{\alpha}(l)+p_{0}^{\alpha} x^{\alpha}(\tau(l)),
$$

which, with (1) and (14), gives

$$
\begin{aligned}
\delta Q(l) z^{\alpha}(\sigma(l)) \leq & q(l) x^{\alpha}(\sigma(l))+q(\tau(l)) p_{0}^{\alpha} x^{\alpha}(\tau(\sigma(l))) \\
\leq & -\left(r(l)\left(z^{\prime \prime}(l)\right)^{\alpha}\right)^{\prime}-\frac{p_{0}^{\alpha}}{\tau_{0}} \\
& \cdot\left(r(\tau(l))\left(z^{\prime \prime}(\tau(l))\right)^{\alpha}\right)^{\prime} .
\end{aligned}
$$

Bringing (13) with $u=\sigma$ and $v=\rho$, and combining it with (16), we get

$$
\begin{aligned}
& \left(r\left(z^{\prime \prime}\right)^{\alpha}+\frac{p_{0}^{\alpha}}{\tau_{0}} r(\tau)\left(z^{\prime \prime}(\tau)\right)^{\alpha}\right)^{\prime}(l) \\
& \quad \leq-\delta Q(l) \Theta_{n}^{\alpha}(\rho(l), \sigma(l)) r(\rho(l))\left(z^{\prime \prime}(\rho(l))\right)^{\alpha} .
\end{aligned}
$$

Secondly, we define $H$ as

$$
H:=r\left(z^{\prime \prime}\right)^{\alpha}+\frac{p_{0}^{\alpha}}{\tau_{0}} r(\tau)\left(z^{\prime \prime}(\tau)\right)^{\alpha}
$$

Since $\left(r(l)\left(z^{\prime \prime}(l)\right)^{\alpha}\right) \leq 0$, we see that

$$
\begin{aligned}
& \left(1+\frac{p_{0}^{\alpha}}{\tau_{0}}\right)\left(r\left(z^{\prime \prime}\right)^{\alpha}\right)(l) \\
& \quad \leq H(l) \leq\left(1+\frac{p_{0}^{\alpha}}{\tau_{0}}\right)\left(r(\tau)\left(z^{\prime \prime}(\tau)\right)^{\alpha}\right)(l) .
\end{aligned}
$$

Therefore,

$$
\frac{\tau_{0}}{\tau_{0}+p_{0}^{\alpha}} H\left(\tau^{-1}(\rho(l))\right) \leq r(\rho(l))\left(z^{\prime \prime}(\rho(l))\right)^{\alpha} .
$$

From (17) and (20), it follows that

$$
H^{\prime}(l)+\frac{\delta \tau_{0}}{\tau_{0}+p_{0}^{\alpha}} Q(l) \Theta_{n}^{\alpha}(\rho(l), \sigma(l)) H\left(\tau^{-1}(\rho(l))\right) \leq 0 .
$$

It is easy to note from (17) that $H^{\prime}(l) \leq 0$ and hence $H\left(\tau^{-1}(\rho(l))\right) \geq H(l)$. Thus, (21) becomes

$$
H^{\prime}(l)+\frac{\delta \tau_{0}}{\tau_{0}+p_{0}^{\alpha}} Q(l) \Theta_{n}^{\alpha}(\rho(l), \sigma(l)) H(l) \leq 0 .
$$

Using the Grönwall inequality, (22) becomes

$$
\begin{aligned}
H\left(\tau^{-1}(u)\right) \geq & H(v) \exp \left(\frac{\delta \tau_{0}}{\tau_{0}+p_{0}^{\alpha}} \int_{\tau^{-1}(u)}^{v}\right. \\
\cdot & \left.Q(h) \Theta_{n}^{\alpha}(\rho(h), \sigma(h)) \mathrm{d} h\right), \text { for } \tau^{1}(u)<v .
\end{aligned}
$$

From (19), we have

$$
\begin{gathered}
z^{\prime \prime}(u) \geq r^{1 / \alpha}(v) z^{\prime \prime}(v)\left(\frac { 1 } { r ( u ) } \operatorname { e x p } \left(\frac{\delta \tau_{0}}{\tau_{0}+p_{0}^{\alpha}} \int_{\tau^{-1}(u)}^{v}\right.\right. \\
\left.\left.\cdot Q(h) \Theta_{n}^{\alpha}(\rho(h), \sigma(h)) \mathrm{d} h\right)\right)^{1 / \alpha} .
\end{gathered}
$$


Integrating (24) twice over $[u, v)$, we get

$$
\begin{gathered}
-z^{\prime}(u) \geq r^{1 / \alpha}(v) z^{\prime \prime}(v) \int_{u}^{v}\left(\frac { 1 } { r ( u _ { 1 } ) } \operatorname { e x p } \left(\frac{\delta \tau_{0}}{\tau_{0}+p_{0}^{\alpha}} \int_{\tau^{-1}\left(u_{1}\right)}^{v}\right.\right. \\
\left.\left.\cdot Q(h) \Theta_{n}^{\alpha}(\rho(h), \sigma(h)) \mathrm{d} h\right)\right)^{1 / \alpha} \mathrm{d} u_{1}, \\
z(u) \geq \Theta_{n+1}(v, u) r^{1 / \alpha}(v) z^{\prime \prime}(v) .
\end{gathered}
$$

This completes the proof.

Theorem 4. Assume that there exists a function $\rho \in C\left(T_{l_{0}}\right.$, $(0, \infty))$ with the properties $\sigma(l)<\rho(l)<\tau(l)$ and $\sigma(l) \leq \tau$ $(\rho(l))$. If

$$
\liminf _{l \rightarrow \infty} \int_{\tau^{-1}(\rho(l))}^{l} Q(h) \Theta_{n}^{\alpha}(\rho(h), \sigma(h)) d h>\frac{\tau_{0}+p_{0}^{\alpha}}{\delta \tau_{0} e},
$$

then $X_{K}=\varnothing$.

Proof. Suppose $x$ belongs to $X_{K}$. As a direct result of Lemma 2 , we get that (8) holds. By following the same approach as in proof of Lemma 2, we get the relationships from (14) to (21). Now, assume $H$ is defined as in (18). From Lemma 1, we have that $z^{\prime \prime}(l)>0$, and hence, $H(l)>0$, for $l \in T_{l_{1}}$. Then, the delay inequality (21) has a positive solution. From Theorem 1 in [22], the associated equation of (21) is

$$
H^{\prime}(l)+\frac{\delta \tau_{0}}{\tau_{0}+p_{0}^{\alpha}} Q(l) \Theta_{n}^{\alpha}(\rho(l), \sigma(l)) H\left(\tau^{-1}(\rho(l))\right)=0,
$$

has also a positive solution. However, it is well known from ([23], Theorem 2) that (34) implies oscillation of (27), a contradiction. This completes the proof.

Example 1. Assume the following NDDE of third order:

$$
\left(x(l)+p_{0} x(a l)\right)^{\prime \prime}+\frac{q_{0}}{l^{3}} x(b l)=0,
$$

where $l \geq 1, a, b \in(0,1)$, and $p_{0}$ and $q_{0}$ are positive. First, we need to calculate $\theta, Q$, and $\Theta_{n}$. Some careful calculations shows that $Q(l)=q_{0} / l^{3}, \theta\left(u_{1}, u_{2}\right)=u_{1}-u_{2}$,

$$
\begin{aligned}
\Theta_{0}\left(u_{1}, u_{2}\right)= & \frac{1}{2}\left(u_{1}-u_{2}\right)^{2}, \\
\Theta_{k}\left(u_{1}, u_{2}\right)= & \frac{1}{1-\mu_{k}} a^{\mu_{k}} u_{1}^{\mu_{k}}\left(\frac{1-\mu_{k}}{2-\mu_{k}} u_{1}^{2-\mu_{k}}\right. \\
& \left.-\left(u_{1}^{1-\mu_{k}} u_{2}-\frac{1}{2-\mu_{k}} u_{2}^{2-\mu_{k}}\right)\right),
\end{aligned}
$$

for $k=1,2, \cdots$, where $c:=(a+b) / 2, \lambda_{0}:=(c-b)^{2} / 2$,

$$
\begin{aligned}
\mu_{k} & :=\frac{a q_{0} \lambda_{k}}{a+p}, \\
\lambda_{k+1} & =\frac{a^{\mu_{k}}}{\mu_{k}-1} c^{\mu_{k}}\left(\frac{1}{\mu_{k}-2} b^{2-\mu_{k}}-\frac{\mu_{k}-1}{\mu_{k}-2} c^{2-\mu_{k}}+b c^{1-\mu_{k}}\right) .
\end{aligned}
$$

Next, if we set $\rho(l)=((a+b) / 2) l$, then applying Theorem 4 requires that

$$
b<\min \left\{a, \frac{a^{2}}{2-a}\right\}=\frac{a^{2}}{2-a} .
$$

Then, it is easy to verify that

$$
\begin{aligned}
\Theta_{0}(c l, b l)= & \frac{1}{2}(c-b)^{2} l^{2}=\lambda_{0} l^{2}, \\
\Theta_{n}(c l, b l)= & \frac{a^{\mu_{n-1}}}{\mu_{n-1}-1} c^{\mu_{n-1}}\left(\frac{1}{\mu_{n-1}-2} b^{2-\mu_{n-1}}\right. \\
& \left.-\frac{\mu_{n-1}-1}{\mu_{n-1}-2} c^{2-\mu_{n-1}}+b c^{1-\mu_{n-1}}\right)=\lambda_{n} l^{2} .
\end{aligned}
$$

Now, we set

$$
H_{n}:=\liminf _{l \rightarrow \infty} \int_{\tau^{-1}(\rho(l))}^{l} Q(h) \Theta_{n}^{\alpha}(\rho(h), \sigma(h)) \mathrm{d} h=q_{0} \lambda_{n} \ln \frac{a}{c} .
$$

In the following particular case,

$$
\left(x(l)+\frac{1}{10} x\left(\frac{9}{10} l\right)\right) "+\frac{811}{10 l^{3}} x\left(\frac{1}{2} l\right)=0,
$$

we note that $H_{0}=0.40864, H_{1}=0.40982$, and

$$
\frac{a+p_{0}}{a \mathrm{e}}=\frac{0.9+0.1}{(0.9) \mathrm{e}}=0.40875 .
$$

Therefore, condition (26) is not satisfied when $n=0$, but satisfies when $n=1$. Thus, (34) has no Kneser solutions.

Remark 5. Very recently, Dzurina et al. ([12], Example 1) proved that (28) is oscillatory if

$$
q_{0}>\max \left\{\frac{8\left(a+p_{0}\right)}{a \mathrm{e}(a-b)^{2} \ln (2 a /(a+b))}, \frac{a+p_{0}}{a b^{2}}\right\} .
$$

In the particular case (34), condition (36) reduces to $81.1>\max \{81.323,4.4444\}=81.323$ (not fulfilled). However, by using our results, the oscillation condition is 81.1 $>80.889$ (fulfilled). Thus, equation (34) is oscillatory. 


\section{Main Results 2: An Improved Approach}

Lemma 6. Assume that $x$ belongs to $X_{N}$ on $\left[l_{1}, \infty\right)$, and $r^{\prime}(l)$ $>0$. Then, $z(l) \geq(1 / 2)\left(l-l_{1}\right) z^{\prime}(l)$ for all $l \geq l_{1}$.

Proof. Assume that $x$ belongs to $X_{N}$ on $\left[l_{1}, \infty\right)$. Then, it follows from Lemma 1 that there is a $l_{1} \geq l_{0}$, such that $z(l)$, $z^{\prime}(l)$, and $z^{\prime \prime}(l)$ are positive and $z^{\prime \prime}$ is nonpositive, for all $l \geq l_{1}$. It is easy to conclude that

$$
\int_{l_{1}}^{l} \int_{l_{1}}^{l} z^{\prime \prime}(s) \mathrm{d} s=z(l)-z\left(l_{1}\right)-z^{\prime}\left(l_{1}\right)\left(l-l_{1}\right),
$$

which, with the fact that $z^{\prime \prime} \leq 0$, gives

$$
\begin{aligned}
& \frac{1}{2}\left(l-l_{1}\right)^{2} z^{\prime \prime}(l) \leq z(l)-z\left(l_{1}\right)-z^{\prime}\left(l_{1}\right)\left(l-l_{1}\right), \\
& z(l) \geq z\left(l_{1}\right)+\left(l-l_{1}\right) z^{\prime}\left(l_{1}\right)+\frac{1}{2}\left(l-l_{2}\right) z^{\prime \prime}(l) .
\end{aligned}
$$

Now, we define

$$
F(l):=\left(l-l_{1}\right) z(l)-\frac{1}{2}\left(l-l_{1}\right)^{2} z^{\prime}(l)
$$

From (39), we get

$$
F^{\prime}(l)=z(l)-\frac{1}{2}\left(l-l_{1}\right)^{2} z^{\prime \prime}(l) \geq z\left(l_{1}\right)+\left(l-l_{1}\right) z^{\prime}\left(l_{1}\right)>0 .
$$

Thus, we have that $F$ is an increasing function with $F\left(l_{1}\right)=0$, and so, $F(l)>0$ for all $l \geq l_{1}$. Therefore, from the definition of $F$, we get the required directly.

Lemma 7. Assume that $x$ belongs to $X_{N}$ on $\left[l_{1}, \infty\right), r^{\prime}(l)>0$, and $p(l) \equiv p$ (constant). Then,

$$
x(l) \geq(1-p) z(l) \sum_{\kappa=0}^{(n-1) / 2} p^{2 \kappa}\left(\frac{\tau^{[2 \kappa+1]}(l)-l_{1}}{l-l_{1}}\right)^{2},
$$

for any odd positive integer $n$ and for all $l \geq l_{2}$, where $l_{2}$ large enough and

$$
\tau^{[0]}:=t, \tau^{[\kappa]}:=\tau\left(\tau^{[\kappa-1]}(t)\right), \text { for } \kappa=1,2, \cdots
$$

Proof. Assume that $x$ belongs to $X_{N}$ on $\left[l_{1}, \infty\right)$. From the definition of $z$, we obtain

$$
x(l)=z(l)-p x(\tau(l))=z(l)-p z(\tau(l))+p^{2} x\left(\tau^{[2]}(l)\right) .
$$

Resuming this procedure, we get, for any $n$ odd positive integers,

$$
\begin{aligned}
x(l) & =\sum_{\kappa=0}^{n}(-1)^{\kappa} p^{\kappa} z\left(\tau^{[\kappa]}(l)\right)+p^{n+1} x\left(\tau^{[n+1]}(l)\right) \\
& \geq \sum_{\kappa=0}^{(n-1) / 2}\left(p^{2 \kappa} z\left(\tau^{[2 \kappa]}(l)\right)-p^{2 \kappa+1} z\left(\tau^{[2 \kappa+1]}(l)\right)\right),
\end{aligned}
$$

for $l \geq l_{1} \geq l_{0}$, where $l_{1}$ is sufficiently large. Since $z^{\prime}(l)>0$ and $\tau^{[2 k+1]}(l) \leq \tau^{[2 k]}(l)$ for all $k=0,1, \cdots,(45)$ becomes

$$
x(l) \geq(1-p) \sum_{\kappa=0}^{(n-1) / 2} p^{2 \kappa} z\left(\tau^{[2 \kappa+1]}(l)\right) .
$$

From Lemma 6, we get $z^{\prime}(l) / z(l) \leq 2 /\left(l-l_{1}\right)$ for all $l \geq l_{1}$. By integrating this inequality from $\tau^{[2 \kappa+1]}(l) \rightarrow l$, we arrive at

$$
z\left(\tau^{[2 \kappa+1]}(l)\right) \geq\left(\frac{\tau^{[2 \kappa+1]}(l)-l_{1}}{l-l_{1}}\right)^{2} z(l),
$$

for all $l \geq l_{2} \geq l_{1}$, which with (46) gives

$$
x(l) \geq(1-p) z(l) \sum_{\kappa=0}^{(n-1) / 2} p^{2 \kappa}\left(\frac{\tau^{[2 \kappa+1]}(l)-l_{1}}{l-l_{1}}\right)^{2} .
$$

This completes the proof.

By replacing the commonly used relationship $x>(1-p) z$ with the new relationship (42), we directly get the following results.

Theorem 8. Assume that $r^{\prime}(l)>0$ and $p(l) \equiv p$ (constant). If

$$
\liminf _{l \rightarrow \infty} \frac{l^{\alpha}}{r(l)} \int_{l}^{\infty} q(s)\left(\frac{\sigma^{2}(s)}{s}\right)^{\alpha} d s>\frac{(2 \alpha)^{\alpha}}{(\alpha+1)^{\alpha+1} p_{*}^{\alpha}},
$$

then $X_{N}=\varnothing$, where

$$
p_{*}:=(1-p) \sum_{\kappa=0}^{(n-1) / 2} p^{2 \kappa}\left(\frac{\tau^{[2 \kappa+1]}(l)-l_{1}}{l-l_{1}}\right)^{2} .
$$

Proof. The proof of this theorem is similar to that of ([7], Corollary 1), and hence, we omit it.

Theorem 9. Assume that $r^{\prime}(l)>0, p(l) \equiv p$ (constant) and $p_{*}$ is defined as (50). If

$$
\begin{gathered}
\underset{l \rightarrow \infty}{\limsup } \int_{l_{0}}^{l}\left(p_{*}^{\alpha} \rho(s) q(s)\left(\frac{\sigma(s)-l_{2}}{s-l_{2}}\right)^{2 \alpha}\right. \\
\left.-\frac{1}{(\alpha+1)^{\alpha+1}} \frac{\left(\rho_{+}^{\prime}(s)\right)^{\alpha+1}}{\rho^{\alpha}(s) \theta^{\alpha}\left(s, l_{2}\right)}\right) d s=\infty,
\end{gathered}
$$

then $X_{N}=\varnothing$. 
Proof. Assume the contrary that $x$ belongs to $X_{N}$ on $\left[l_{1}, \infty\right)$. From Lemma 7, we get that (42) holds. Combining (1) and (42), we find

$$
\left(r(l)\left(z^{\prime \prime}(l)\right)^{\alpha}\right)^{\prime} \leq-p_{*}^{\alpha} q(l) z^{\alpha}(\sigma(l))
$$

for $l \geq l_{2} \geq l_{1}$. Now, we define

$$
w(l):=\rho(l) \frac{r(l)\left(z^{\prime \prime}(l)\right)^{\alpha}}{z^{\alpha}(l)}
$$

Then, $w(l)>0$ for all $l \geq l_{1}$, and

$$
\begin{aligned}
w^{\prime}(l)= & \frac{\rho^{\prime}(l)}{\rho(l)} w(l)+\rho(l) \frac{\left(r(l)\left(z^{\prime \prime}(l)\right)^{\alpha}\right)^{\prime}}{z^{\alpha}(l)} \\
& -\alpha \rho(l) \frac{r(l)\left(z^{\prime \prime}(l)\right)^{\alpha}}{z^{\alpha+1}(l)} z^{\prime}(l) .
\end{aligned}
$$

From Lemma 6, we get $z^{\prime}(l) / z(l) \leq 2 /\left(l-l_{2}\right)$ for all $l \geq l_{2}$. By integrating this inequality from $\sigma(l) \rightarrow l$, we arrive at

$$
z(\sigma(l)) \geq\left(\frac{\sigma(l)-l_{2}}{l-l_{2}}\right)^{2} z(l)
$$

for $l \geq l_{3} \geq l_{2}$. Since $\left(r\left(z^{\prime \prime}\right)^{\alpha}\right)^{\prime} \leq 0$, we have

$$
z^{\prime}(l) \geq \int_{l_{2}}^{l} \frac{1}{r^{1 / \alpha}(s)} r^{1 / \alpha}(s) z^{\prime \prime}(s) \mathrm{d} s \geq r^{1 / \alpha}(l) z^{\prime \prime}(l) \theta\left(l, l_{2}\right)
$$

From (52)-(56), we obtain

$$
\begin{aligned}
w^{\prime}(l) \leq & \frac{\rho_{+}^{\prime}(l)}{\rho(l)} w(l)-p_{*}^{\alpha} \rho(l) q(l)\left(\frac{\sigma(l)-l_{2}}{l-l_{2}}\right)^{2 \alpha} \\
& -\alpha \frac{\theta\left(l, l_{2}\right)}{\rho^{1 / \alpha}(l)} w^{1+1 / \alpha}(l) .
\end{aligned}
$$

Next, we define

$$
\Phi(w)=\frac{\rho_{+}^{\prime}}{\rho} w-\alpha \frac{\theta}{\rho^{1 / \alpha}} w^{1+1 / \alpha}
$$

Then, $\Phi^{\prime}(w)=\rho_{+}^{\prime} / \rho-(\alpha+1) \rho^{-1 / \alpha} \theta w^{1 / \alpha}$, and so $\Phi$ attains its maximum value on $\mathbb{R}$ at $w^{*}=\rho\left(\rho_{+}^{\prime} /((\alpha+1) \rho \theta)\right)^{\alpha}$ and

$$
\Phi(s) \leq \max _{w \in \mathbb{R}} \Phi(s)=\frac{1}{(\alpha+1)^{\alpha+1}} \frac{\left(\rho_{+}^{\prime}\right)^{\alpha+1}}{\rho^{\alpha} \theta^{\alpha}} .
$$

Combining (57) and (59), we find

$w^{\prime}(l) \leq-p_{*}^{\alpha} \rho(l) q(l)\left(\frac{\sigma(l)-l_{2}}{l-l_{2}}\right)^{2 \alpha}+\frac{1}{(\alpha+1)^{\alpha+1}} \frac{\left(\rho_{+}^{\prime}(l)\right)^{\alpha+1}}{\rho^{\alpha}(l) \theta^{\alpha}\left(l, l_{2}\right)}$.

By integrating this inequality from $l_{3} \rightarrow l$, we arrive at

$$
\begin{aligned}
& \int_{l_{3}}^{l}\left(p_{*}^{\alpha} \rho(s) q(s)\left(\frac{\sigma(s)-l_{2}}{s-l_{2}}\right)^{2 \alpha}-\frac{1}{(\alpha+1)^{\alpha+1}} \frac{\left(\rho_{+}^{\prime}(s)\right)^{\alpha+1}}{\rho^{\alpha}(s) \theta^{\alpha}\left(s, l_{2}\right)}\right) \mathrm{d} s \\
& \quad \leq w\left(l_{1}\right),
\end{aligned}
$$

which contradicts (51). This contradiction completes the proof.

Theorem 10. Assume that $r^{\prime}(l)>0, p(l) \equiv p$ (constant), and $p_{*}$ is defined as (50). If

$$
Y^{\prime}(l)+q(l)\left(p_{*} \int_{l_{2}}^{\sigma(l)} \theta\left(s, l_{2}\right) d s\right)^{\alpha} Y(\sigma(l))=0,
$$

is oscillatory, then $X_{N}=\varnothing$.

Proof. Assume the contrary that $x$ belongs to $X_{N}$ on $\left[l_{1}, \infty\right)$. Proceeding as in the proof of Theorem 9, we obtain (52) and (56) hold for $l \geq l_{2} \geq l_{1}$. Integrating (56) from $l_{2} \rightarrow l$ and taking into account the fact that $\left(r(l)\left(z^{\prime \prime}(l)\right)^{\alpha}\right)^{\prime} \leq 0$, we get

$$
z(l) \geq r^{1 / \alpha}(l) z^{\prime \prime}(l) \int_{l_{2}}^{l} \theta\left(s, l_{2}\right) \mathrm{d} s,
$$

which with (52) gives

$$
\begin{aligned}
& \left(r(l)\left(z^{\prime \prime}(l)\right)^{\alpha}\right)^{\prime}+q(l)\left(p_{*} \int_{l_{2}}^{\sigma(l)} \theta\left(s, l_{2}\right) \mathrm{d} s\right)^{\alpha} r(\sigma(l)) \\
& \cdot\left(z^{\prime \prime}(\sigma(l))\right)^{\alpha} \leq 0 .
\end{aligned}
$$

Thus, if we set $Y:=r\left(z^{\prime \prime}\right)^{\alpha}>0$, then we note that $Y$ is a positive solution of the differential inequality

$$
Y^{\prime}(l)+q(l)\left(p_{*} \int_{l_{2}}^{\sigma(l)} \theta\left(s, l_{2}\right) \mathrm{d} s\right)^{\alpha} Y(\sigma(l)) \leq 0 .
$$

Thus, it follows from ([22], Theorem 1) that the corresponding equation (62) also has a positive solution, which is a contradiction. This contradiction completes the proof. 
Table 1: Criteria that ensure that $X_{N}=\varnothing$ for (66).

\begin{tabular}{ccc}
\hline \multicolumn{3}{c}{ Hille and Nehari type criterion } \\
$C_{1}$ & Corollary 1 in [7] & $q_{0}>6912.0$ \\
$C_{2}$ & Condition (68) & $q_{0}>3266.8$ \\
\hline \multicolumn{3}{c}{ Using comparison theorems with 1 $1^{\text {sl }}$-order DDE } \\
$C_{3}$ & Theorem 2.4 in [8] & $q_{0}>1491.0$ \\
$C_{4}$ & Condition (70) & $q_{0}>704.69$ \\
\hline
\end{tabular}

Example 2. Consider the third-order NDDE,

$$
\left.\left(l\left[\left(x(l)+\frac{3}{4} x\left(\frac{9}{10} l\right)\right)^{\prime \prime}\right]^{3}\right)\right)^{\prime}+\frac{q_{0}}{l^{6}} x^{3}\left(\frac{1}{2} l\right)=0
$$

where $l>0$ and $q_{0}>0$. Then, it is easy to verify that

$$
\begin{aligned}
\tau^{[2 \kappa+1]}(l) & =\left(\frac{9}{10}\right)^{2 \kappa+1} l \\
p_{*} & :=\frac{1}{4} \sum_{\kappa=0}^{(n-1) / 2}\left(\frac{3}{4}\right)^{2 \kappa}\left(\frac{9}{10}\right)^{4 \kappa+2} .
\end{aligned}
$$

To apply Theorem 8, condition (49) reduces to

$$
\begin{aligned}
& \liminf _{l \rightarrow \infty} \frac{l^{\alpha}}{r(l)} \int_{l}^{\infty} q(s)\left(\frac{\sigma^{2}(s)}{s}\right)^{\alpha} \mathrm{d} s \\
& \quad=\liminf _{l \rightarrow \infty} l^{2} \int_{l}^{\infty} \frac{q_{0}}{s^{6}}\left(\frac{1}{4} s\right)^{3} \mathrm{~d} s=q_{0} \frac{1}{2}\left(\frac{1}{4}\right)^{3}>\frac{6^{3}}{2^{8} p_{*}^{3}} .
\end{aligned}
$$

On the other hand, using Theorem 10, the class $X_{N}$ of (66) is empty if the first-order DDE

$$
Y^{\prime}(l)+\frac{2 q_{0}}{l}\left(\frac{9}{40} p_{*}\right)^{3} Y\left(\frac{1}{2} l\right)=0,
$$

is oscillatory. Based on Theorem 2.1 in [24], the first-order DDE (69) is oscillatory if

$$
\liminf _{l \rightarrow \infty} \int_{l / 2}^{l} 2 q_{0}\left(\frac{9}{40} p_{*}\right)^{3} \frac{1}{s} d s=2 q_{0}\left(\frac{9}{40} p_{*}\right)^{3} \ln 2>\frac{1}{\mathrm{e}}
$$

Remark 11. In order to ensure that the class $X_{N}$ of (66) is empty, Table 1 compares between our criteria and the previous related one:

It is easy to notice that Theorem 10 supports the most efficient condition. Thus, our results improve the results in $[7,8]$.

\section{Conclusion}

Most studies investigating the asymptotic behavior of solutions of DDEs provided only sufficient criteria. This was a major motivation for the multiplicity of methods and techniques used in the study, so that a larger area of models could be covered. The aim of this study is to develop new criteria that have an iterative nature, so that we can apply them when the other criteria fail. First, we established a criterion to ensure that there are no Kneser solutions of (1) based on the comparison with a first-order DDE. Further, we obtained various criteria ensuring that $X_{N}$ is empty. By combining the results in Sections 2 and 3, we can obtain new conditions for the oscillation of all solutions of (1). It would be interesting to extend our results to the NDDE

$$
\left(r_{2}(l)\left(\left(r_{1}(l)\left(z^{\prime}(l)\right)^{\alpha_{1}}\right)^{\prime}\right)^{\alpha_{2}}\right)^{\prime}+q(l) f(x(\sigma(l)))=0 .
$$

It is also interesting to eliminate some restrictions that are usually imposed on the coefficients of the NDDEs studied.

\section{Data Availability}

No data were used to support this study.

\section{Conflicts of Interest}

There are no competing interests.

\section{Acknowledgments}

This study was supported by the Taif University Researchers Supporting Project number (TURSP-2020/20), Taif University, Taif, Saudi Arabia.

\section{References}

[1] M. A. Alqudah, C. Ravichandran, T. Abdeljawad, and N. Valliammal, "New results on Caputo fractional-order neutral differential inclusions without compactness," Advances in Difference Equations, vol. 2019, no. 1, Article ID 528, 2019.

[2] D. D. Bainov and D. P. Mishev, Oscillation Theory for Neutral Differential Equations with Delay, CRC Press, 1991.

[3] J. K. Hale, Functional Differential Equations, in Analytic Theory of Differential Equations, Springer, 1971.

[4] K. Logeswari and C. Ravichandran, "A new exploration on existence of fractional neutral integro- differential equations in the concept of Atangana-Baleanu derivative," Physica A: Statistical Mechanics and its Applications, vol. 544, article 123454, 2020.

[5] C. Ravichandran, N. Valliammal, and J. J. Nieto, "New results on exact controllability of a class of fractional neutral integrodifferential systems with state-dependent delay in Banach spaces," Journal of the Franklin Institute, vol. 356, no. 3, pp. 1535-1565, 2019.

[6] N. Valliammal and C. Ravichandran, "Results on fractional neutral integro-differential systems with state-dependent delay in Banach spaces," Nonlinear Studies, vol. 25, no. 1, pp. 159171, 2018.

[7] B. Baculikova and J. Dzurina, "Oscillation of third-order neutral differential equations," Mathematical and Computer Modelling, vol. 52, no. 1-2, pp. 215-226, 2010.

[8] B. Baculikova and J. Dzurina, "On the asymptotic behavior of a class of third order nonlinear neutral differential equations," 
Central European Journal of Mathematics, vol. 8, no. 6, pp. 1091-1103, 2010.

[9] T. Candan, "Asymptotic properties of solutions of third-order nonlinear neutral dynamic equations," Advances in Difference Equations, vol. 2014, no. 1, Article ID 35, 2014.

[10] G. E. Chatzarakis, J. Dzurina, and I. Jadlovska, "Oscillatory and asymptotic properties of third-order quasilinear delay differential equations," Journal of Inequalities and Applications, vol. 2019, no. 1, Article ID 23, 2019.

[11] G. E. Chatzarakis, S. R. Grace, and I. Jadlovska, "Oscillation criteria for third-order delay differential equations," Advances in Difference Equations, vol. 2017, no. 1, Article ID 330, 2017.

[12] J. Dzurina, S. R. Grace, and I. Jadlovska, "On nonexistence of Kneser solutions of third-order neutral delay differential equations," Applied Mathematics Letters, vol. 88, pp. 193-200, 2019.

[13] E. M. Elabbasy, T. S. Hassan, and B. M. Elmatary, "Oscillation criteria for third order delay nonlinear differential equations," Electronic Journal of Qualitative Theory of Differential Equations, vol. 2012, no. 5, pp. 1-9, 2012.

[14] T. Li, C. Zhang, and G. Xing, "Oscillation of third-order neutral delay differential equations," Abstract and Applied Analysis, vol. 2012, Article ID 569201, 11 pages, 2012.

[15] O. Moaaz, D. Chalishajar, and O. Bazighifan, "Asymptotic behavior of solutions of the third order nonlinear mixed type neutral differential equations," Mathematics, vol. 8, no. 4, p. $485,2020$.

[16] E. Thandapani and T. Li, "On the oscillation of third-order quasi-linear neutral functional differential equations," Archiv der Mathematik, vol. 47, pp. 181-199, 2011.

[17] B. Baculikova and J. Dzurina, "On the oscillation of odd order advanced differential equations," Boundary Value Problems, vol. 2014, no. 1, Article ID 214, 2014.

[18] O. Moaaz, "Oscillatory behavior of solutions of odd-order nonlinear delay differential equations," Advances in Difference Equations, vol. 2020, no. 1, Article ID 357, 2020.

[19] O. Moaaz, J. Awrejcewicz, and A. Muhib, "Establishing new criteria for oscillation of odd-order nonlinear differential equations," Mathematics, vol. 8, no. 6, p. 937, 2020.

[20] O. Moaaz, D. Baleanu, and A. Muhib, "New aspects for nonexistence of Kneser solutions of neutral differential equations with odd-order," Mathematics, vol. 8, no. 4, p. 494, 2020.

[21] I. Kiguradze and T. Chanturia, Asymptotic Properties of Solutions of Nonautonomous Ordinary Differential Equations, vol. 89 of Mathematics and Its Applications (Soviet Series), Kluwer Academic Publishers Group, DordrechtTranslated from the 1985 Russian original 1993.

[22] C. Philos, "On the existence of nonoscillatory solutions tending to zero at $\infty$ for differential equations with positive delays," Archiv der Mathematik, vol. 36, no. 1, pp. 168-178, 1981.

[23] Y. Kitamura and T. Kusano, "Oscillation of first-order nonlinear differential equations with deviating arguments," Proceedings of the American Mathematical Society, vol. 78, no. 1, pp. 64-68, 1980.

[24] G. S. Ladde, V. Lakshmikantham, and B. G. Zhang, Oscillation Theory of Differential Equations with Deviating Arguments, Monogr. Textbooks Pure Applied Mathathematics, Marcel Dekker, New York, 1987. 